\title{
Heart failure presentation thirty years after tricuspid valvectomy for infective endocarditis
}

\author{
Michael P. Rogers, MD, MS, ${ }^{a}$ Christopher Bitetzakis, DO, ${ }^{\mathrm{b}}$ Aarti Patel, MD, ${ }^{\mathrm{b}}$ and Robert L. Hooker, MD,
} Tampa, Fla, and Tucson, Ariz

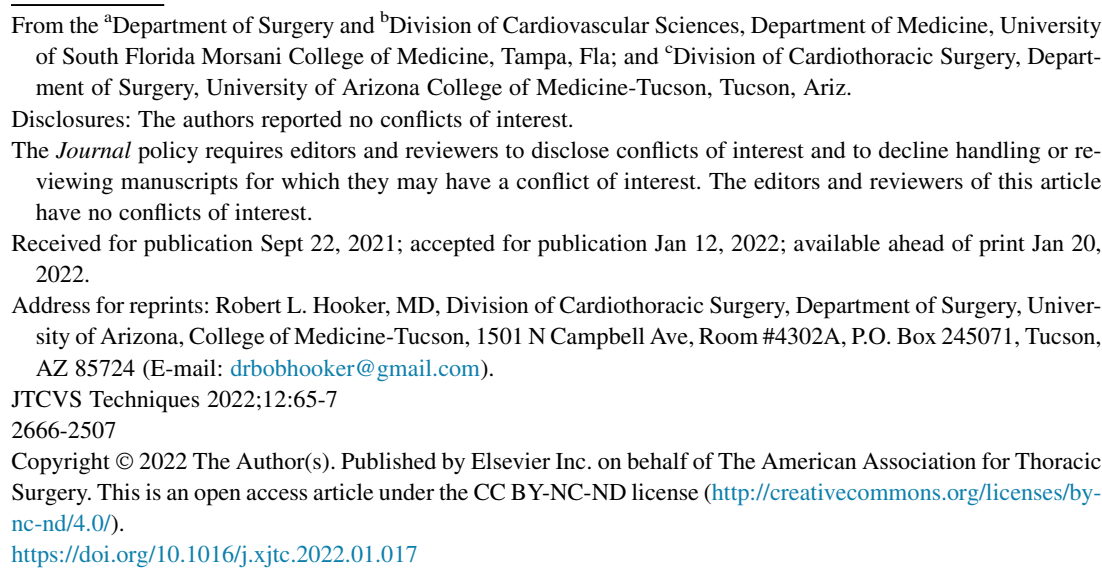

The incidence of tricuspid valve infective endocarditis secondary to intravenous (IV) drug abuse has risen sharply during the opioid epidemic in the United States. ${ }^{1}$ Although medical management with long-term IV antibiotics is the primary treatment strategy, several patients may not respond to treatment or have residual tricuspid insufficiency requiring operative intervention. ${ }^{1}$ The optimal surgical management of tricuspid valve endocarditis has evolved over the last several decades and has included valvectomy, valve repair, and valve replacement. ${ }^{1,2}$ We report the follow-up and management of a long-term survivor who had previously undergone tricuspid valvectomy for infective endocarditis 30 years previously.

\section{CASE PRESENTATION}

A 63-year-old man with history of hypertension, diabetes mellitus, hepatomegaly, and IV drug abuse presented to our clinic in June 2016 with complaints of increasing dyspnea over the last several months, orthopnea, and lower-extremity edema. He developed tricuspid valve infective endocarditis requiring tricuspid valvectomy at an outside institution in June 1986 (30-year history) and was subsequently lost to follow-up. The patient notably had stopped using drugs and led a productive life. Despite his social situation, the patient was adherent with prescribed labetalol, lisinopril, metformin, and sliding-scale insulin. He initially underwent workup at our institution with transthoracic echocardiography, which demonstrated absence of the tricuspid valve with torrential regurgitation and reversal of flow in the inferior vena cava with severe right ventricular (RV) dilatation (RV internal dimension in diastole $6.13 \mathrm{~cm}$ ) and moderate $\mathrm{RV}$ dysfunction (Figure 1). Subsequent cardiac catheterization demonstrated

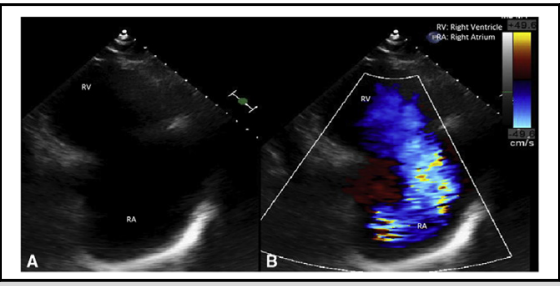

Surgically absent tricuspid valve $(A)$ with torrential regurgitation (B).

CENTRAL MESSAGE

Patients who undergo tricuspid

valvectomy may develop

symptoms of right-sided heart

failure and require operative

reintervention with valve

replacement.

See Commentary on page 68. elevated right heart pressures (right atrium $21 \mathrm{~mm} \mathrm{Hg}, \mathrm{RV}$ $50 / 20 \mathrm{~mm} \mathrm{Hg}$ ) with ventricularized waveform. The patient was discussed at a multidisciplinary heart valve conference and deemed appropriate for tricuspid replacement.

Intraoperative transesophageal echocardiography again demonstrated torrential tricuspid regurgitation with dilatation of the right atrium and ventricle. The starting central venous pressure was $26 \mathrm{~mm} \mathrm{Hg}$. After sternal reentry, a "beating-heart" strategy for replacement was employed. A $33-\mathrm{mm}$ pericardial valve was placed with no change in the electrocardiogram during the procedure. The patient was weaned from cardiopulmonary bypass uneventfully. Postprocedure transesophageal echocardiography demonstrated a normally functioning tricuspid bioprosthesis without regurgitation. The patient was subsequently discharged on postoperative day 9 after treatment of postoperative tracheobronchitis. Transthoracic echocardiography before discharge again demonstrated satisfactory replacement without significant insufficiency and decreased RV systolic pressure (37 mm Hg) (Figure 2). Recent follow-up transthoracic echocardiography again demonstrated normal tricuspid prosthetic valve function, normalized estimated RV systolic pressure, and RV remodeling without residual dilatation. 


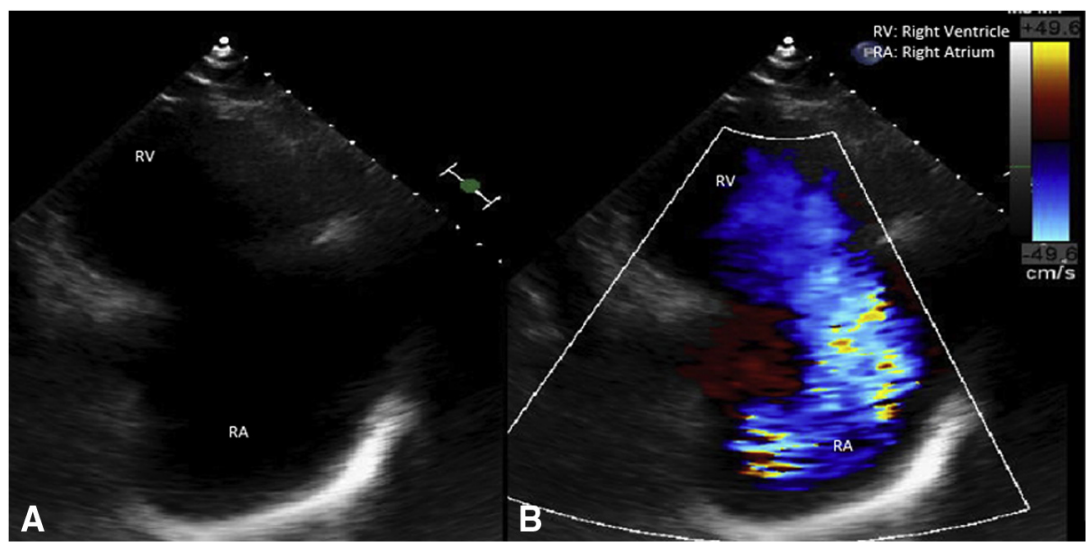

FIGURE 1. A, Transthoracic echocardiogram parasternal right ventricular inflow view with surgically absent tricuspid valve. B, Right ventricular inflow view in systole with color Doppler revealing torrential regurgitation involving the entirety of the right atrium.

\section{COMMENT}

Surgical intervention for infective endocarditis is indicated in patients who do not respond to initial IV antibiotic treatment, develop heart failure, have embolic events, or residual tricuspid insufficiency. ${ }^{1,3}$ However, debate over the best surgical intervention for right-sided endocarditis has persisted over the last several decades and included valve repair, replacement, and valvectomy. Recent analyses and consensus guidelines from the American Association for Thoracic Surgery recommend vegetation debridement and repair of the valve when possible, although recent studies suggest replacement remains the most commonly performed procedure. ${ }^{4,5}$

Given concern for the high rates of recidivism and lack of social and financial support in this patient population, tricuspid valvectomy with delayed replacement until completion of a drug treatment program had remained a practical option in this cohort. While previous reports have demonstrated long-term survival of patients without a tricuspid valve, particularly in those with normal pulmonary artery hemodynamics, greater than $25 \%$ of these patients are not able to tolerate tricuspid insufficiency and require subsequent replacement. In addition, this cohort may often be difficult to follow clinically, given high recidivism and difficulty of follow-up. We report what we believe to be the lengthiest delay to tricuspid valve replacement following tricuspid valvectomy (30 years) for IV drug abuse after loss to follow-up in the literature.

Optimal surgical management is paramount to ensuring superior outcomes in this cohort. Protos and colleagues' recent single-center review ${ }^{2}$ comparing tricuspid valvectomy, repair, and replacement demonstrated similar perioperative outcomes between treatment strategies, however with more patients in the replacement group experiencing unplanned readmissions stemming from infection of their prosthetic valve. Additional analysis of the Society of Thoracic Surgeons National Database by this group revealed valvectomy as an independent predictor of operative mortality and recommended repair of the native valve whenever anatomically possible to avoid recurrent valve

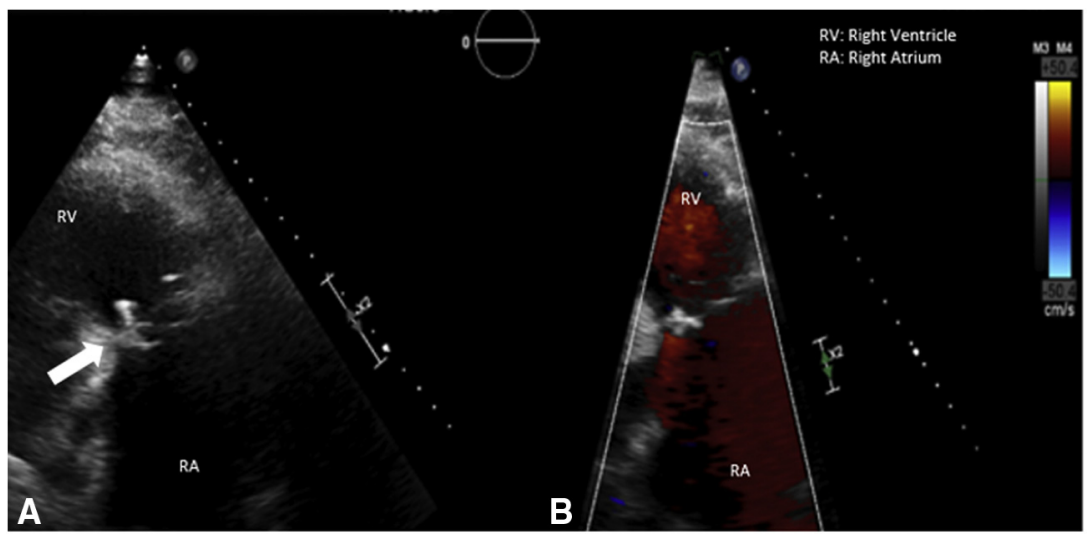

FIGURE 2. A, Transthoracic echocardiogram parasternal right ventricular inflow view with a well-seated bioprosthetic tricuspid valve (arrow). B, Right ventricular inflow view in systole with color Doppler confirming no significant regurgitation. 
infection and prosthetic valve degeneration. ${ }^{1}$ In addition, considerations for preserving RV function should be made when contemplating operative timing.

The surgical treatment of right-sided infective endocarditis has changed over the past several decades. Contemporary treatment guidelines recommend preservation of the native valve whenever possible. Symptomatic patients with previous tricuspid resection should undergo replacement when feasible.

\section{References}

1. Slaughter MS, Badhwar V, Ising M, Ganzel BL, Sell-Dottin K, Jawitz OK, et al. Optimum surgical treatment for tricuspid valve infective endocarditis: an analysis of the Society of Thoracic Surgeons National database. J Thorac Cardiovasc Surg. 2021;161:1227-35.e1.

2. Protos AN, Trivedi JR, Whited WM, Rogers MP, Owolabi U, Grubb KJ, et al Valvectomy versus replacement for the surgical treatment of tricuspid endocarditis. Ann Thorac Surg. 2018;106:664-9.

3. Nath J, Foster E, Heidenreich PA. Impact of tricuspid regurgitation on long-term survival. J Am Coll Cardiol. 2004;43:405-9.

4. AATS Surgical Treatment of Infective Endocarditis Consensus Guidelines Writing Committee Chairs, Pettersson GB, Coselli JS, Hussain ST, Griffin B, Blackstone EH, et al. 2016 The American Association for Thoracic Surgery (AATS) consensus guidelines: surgical treatment of infective endocarditis: executive summary. J Thorac Cardiovasc Surg. 2017;153: 1241-58.e29.

5. Wallen TJ, Szeto W, Williams M, Atluri P, Arnaoutakis G, Fults M, et al Tricuspid valve endocarditis in the era of the opioid epidemic. $J$ Card Surg. 2018;33:260-4. 